\title{
Die demokratische Gleichheit des Loses: Aus der Nische des Rechtswesens zurück in die Polis
}

\author{
Tamara Ehs ${ }^{*}$
}

\section{Zusammenfassung}

Das Losverfahren, ein Klassiker der politischen Ideengeschichte, kommt seit einiger Zeit wieder zum Einsatz. Überall dort, wo die repräsentative Demokratie eine Vertrauenskrise erlebt, treten vermehrt geloste Bürgerräte auf den Plan. Sie sollen Menschen in den politischen Prozess bringen, die nicht schon von selbst AktivbürgerInnen sind, und eine nicht-populistische Maßnahme zur Partizipation bieten. Ausgehend von einem ideengeschichtlichen und historischen Überblick erläutert der Beitrag, wie das Losverfahren während der Entwicklung moderner Staaten allein im Rechtswesen überdauerte, nun aber angesichts der Demokratiekrise wiederentdeckt und befördert wird, um mehr demokratische Gleichheit und höhere Legitimation politischer Entscheidungen zu erlangen.

Schlagwörter: Bürgerrat, Demokratie, Gleichheit, Losverfahren, Partizipation

The democratic equality of the lot: from the niche of the legal system back to the polis Abstract

The political lottery (sortition), a classic in the history of political ideas, is now again in use. Wherever representative democracy is confronted with a crisis of confidence, more and more citizens' assemblies or councils drafted by lot appear on the scene. They are supposed to bring people into the political process who are not already active citizens by themselves, offering a non-populist measure of participation. Starting from a historical overview, the article explains how the lottery process outlasted in the legal system alone during the development of the modern state, but is now being rediscovered and promoted in the face of the democratic crisis in order to achieve more equality and egalitarianism, and even higher legitimacy for political decisions.

Keywords: Citizens' Assembly, Democracy, Equality, Sortition, Participation

${ }^{*}$ Tamara Ehs, Vorsitzende IG Demokratie sowie Projektleiterin am Institut für Politikwissenschaft der Universität Wien. E-Mail: tamara.ehs@univie.ac.at 


\section{Einleitung}

Ob nun die irische Citizens' Assembly, die Vorarlberger Bürgerräte oder neuerdings Mitgliederräte in Parteien - was unter Headlines wie „innovativer Prozess“ oder gar „radikales Demokratieexperiment“ seit einigen Jahren als mögliches Heilmittel gegen die Krise der Demokratie zur Anwendung kommt, ist für die politische Ideengeschichte ein Klassiker: der Einsatz des Loses. Bereits Aristoteles meinte, Wahlen seien aufgrund des Reichtums oligarchisch und aufgrund der Tüchtigkeit aristokratisch; einzig das Los verbürge Demokratie (Aristoteles 1991).

Der vorliegende Beitrag thematisiert das Revival des Losverfahrens. Unter der Prämisse demokratischer Gleichheit, die den Einbezug aller BürgerInnen in den politischen Prozess befördern soll, kommen in jüngster Zeit vermehrt geloste Bürgerräte zum Einsatz. Mittels qualifizierter Zufallsauswahl stellen sie ein Deliberationsorgan dar, das die Bevölkerung abbildet. Auf diese Weise werden nicht nur Menschen an der Politik beteiligt, die sonst aufgrund ihres sozioökonomischen Status, ihres Bildungsgrades oder ihrer Herkunft ungehört bleiben, sondern man verspricht sich davon auch eine höhere Input-Legitimation politischer Entscheidungen, mehr Systemvertrauen und durch die Demokratisierung des Lobbying auch eine höhere Output-Legitimation. Das aleatorische Instrument soll als Antwort auf die vielen zeitgenössischen Rufe nach "mehr“ und „echter“ Demokratie - worunter meist direktdemokratische Instrumente und die Volksgesetzgebung gemeint sind (Ehs/Willroider 2013) - die repräsentative Demokratie allerdings nicht ersetzen, sondern stärken.

Ausgehend von einem ideengeschichtlichen und historischen Überblick erläutert der Text, wie das Losverfahren während der Entwicklung moderner Staatlichkeit allein im Rechtswesen (und zwar in der Laiengerichtsbarkeit) überdauerte, nun aber angesichts multipler Krisen und einer neuen Sozialen Frage für den Bereich der Gesetzgebung wiederentdeckt wird. Die darauf folgende demokratiepolitische Analyse stellt auf Fragen der (Un-)Gleichheit ab und verhandelt aleatorische Maßnahmen als nicht-populistische Antwort auf eine defizitäre Demokratie.

\section{Historischer Überblick}

Um politische Amtsträger zu bestimmen, wurde bereits in der athenischen Polis nicht bewusst ausgewählt, sondern überwiegend per Zufall entschieden (Taylor 2007). In der Neuzeit verwendeten etwa Florenz und Venedig aleatorische Verfahren zur Besetzung der höchsten Ämter. ${ }^{1}$ Damals bestätigte Montesquieu in De lesprit des lois (1748) eine Erkenntnis, die Aristoteles schon zweitausend Jahre zuvor gewonnen hatte: „Wahl durch Los [le suffrage par le sort] entspricht der Natur der Demokratie, Wahl durch Abstimmung [le suffrage par choix] der Natur der Aristokratie." JeanJacques Rousseau pflichtete im Gesellschaftsvertrag bei und sprach sich hinsichtlich moderner Staatlichkeit für eine Kombination von Wahl und Los aus, um einerseits Inkompetenz zu vermeiden, andererseits demokratische Gleichheit zu garantieren: „Wenn Wahl und Los gemischt werden, muss erstere dazu dienen, Stellen zu besetzen, die besondere Fähigkeiten verlangen, zum Beispiel militärische Posten. Das Los eignet sich dagegen bei Stellen, wo gesunder Menschenverstand, Gerechtigkeitssinn und Redlichkeit ausreichen, wie bei richterlichen Ämtern“ (Rousseau 1762: 476f.).

Der Sinn und Zweck der politischen Lotterie lag demnach darin, die Bürde und Würde eines politischen Amtes unter allen Bürgern ${ }^{2}$ gleichmäßig zu verteilen, also die politische Gleichheit zu befördern. Niemand sollte sich aus Eitelkeit und Gewinnsucht für ein Amt bewerben, niemand sollte durch die Möglichkeit einer Wiederwahl korrumpierte Entscheidungen treffen. So viele Bürger wie möglich sollten im Rotationsprinzip Teil von Legislative, Exekutive und Judikative sein, worin sich nicht nur John Rawls' zeitlose Gerechtigkeitstheorie (1971) wiederfindet, sondern was ebenso dem antiken und bis heute beschworenen Ideal der Demokratie entspricht: Identität von Herrschern und Beherrschten.

Doch obwohl die Aufklärer eben erst das aleatorische Rekrutierungsverfahren gepriesen hatten, beruhte die Entwicklung der modernen Staaten bald darauf sowohl im revolutionären Frankreich als auch in den USA auf Wahlen, wie der Politologe Bernard Manin (2007: 112) erstaunt feststellte: „Zur gleichen Zeit, als die Gründerväter die Gleichheit aller Bürger deklarierten, entschied man sich auf beiden Seiten des Atlantiks ohne die geringsten Bedenken für eine unein-

1 Zur Geschichte des Losverfahrens siehe allen voran Buchstein 2009 .

2 Ich bezeichne hier historisch korrekt nur das männliche Geschlecht und schreibe explizit „Bürger“, um auf den Ausschluss von Normunterworfenen ohne Bürgerrechte (wie Frauen und Sklaven) hinzuweisen. 
geschränkte Herrschaft einer Auswahlmethode, die seit langem als aristokratisch galt.“

David Van Reybrouck ging in seinem provokant Gegen Wahlen betitelten Buch dieser Geschichte nach und kam einer ziemlich elitären Auffassung von „Volk“ und „Demokratie“ auf die Spur: Sowohl die Vertreter der Französischen als auch der Amerikanischen Revolution mieden gar das Wort „Demokratie“ oder verwendeten es bloß, um es mit Chaos und Extremismus gleichzusetzen (Van Reybrouck 2016: 88ff.). Daher sollte die Führerauslese per Wahl erfolgen; und auch wenn das aktive Wahlrecht gewissen, wenn auch unter heutigen Vorzeichen eingeschränkten Gleichheitsmaßstäben genügte, war das passive Wahlrecht in praxi nicht gleich, wie James Madison in den Federalist Papers (1787: 97) festhielt: „Wer kommt als Objekt einer Wahl durch das Volk in Betracht? Jeder Bürger, dessen Verdienste ihn der Achtung und dem Vertrauen seines Landes empfehlen." Auch in Frankreich und ebenso in England setzte man forthin auf Wahlen und bei der Auslese der politischen Amtsträger auf virtue und wisdom, wie es bei Edmund Burke (1790) hieß. Wer sich „um ein Amt verdient“ gemacht hatte, wer „Tugend“ und „Weisheit“ für die Politik besaß, war gewiss nicht der Pöbel, der gemeine Mann und schon gar nicht die gemeine Frau. Das Volk hatte zwar seine ErbmonarchInnen vertrieben, bekam sie aber lediglich durch gewählte Oligarchen und Aristokraten ersetzt. Aufgrund der fehlenden sozialen Durchlässigkeit oder gar dem Ende der Klassengesellschaft war politische Macht weiterhin nur einer kleinen Elite von Wohlhabenden und Höhergebildeten zugänglich.

Das änderte sich erst im späten 19. und vor allem im 20. Jahrhundert. Das Wahlrecht erfuhr Schritt für Schritt seine Ausweitung und mittels Massenparteien und Gewerkschaftsbewegung eroberten allmählich auch ArbeiterInnen das Parlament und politische Führungspositionen. Die Einbindung nun möglichst aller sozialen Milieus und Schichten stärkte die repräsentative Demokratie, die Wahlbeteiligung war meist sehr hoch und zudem über alle Bevölkerungsschichten weitgehend gleich verteilt (Franklin 2004). Der Wohlfahrtsstaat westeuropäischen Zuschnitts stützte außerdem das Gleichheitsversprechen als die eigentliche integrative Sozialerzählung des Kapitalismus und während „les trente glorieuses“ (Rilling 2014) bildete sich der Zeitgeist vom „Ende der Geschichte“, zu dem auch die auf Wahlen beruhende Demokratie zählte. Über das Losverfahren oder gar Alternativen zum Parlamentarismus dachten damals bis auf einige Ausnahmen wie
Robert Dahl (1971) und John Burnheim (1987) nur sehr wenige nach.

\section{Losverfahren im Rechtsystem: Laienge- richtsbarkeit}

War das aleatorische System im Zusammenhang mit politischer Ämtervergabe außer für ein paar HistorikerInnen in Vergessenheit geraten, so hatte es allerdings im Rechtswesen überlebt. In der Laiengerichtsbarkeit wurden und werden bis heute Schöffen und Geschworene sowohl im angelsächsischen als auch im kontinentalen Rechtsraum - ungeachtet ihrer verschiedenen Zuständigkeiten - per Zufall ernannt (Duxbury 1999). In Österreich wurde die Laiengerichtsbarkeit im Jahre 1873 eingeführt und blieb mit Unterbrechungen trotz oftmaliger Kritik erhalten. Heute regelt das Bundesgesetz vom 25. April 1990 über die Berufung der Geschworenen und Schöffen (GSchG, BGBl. Nr. 256/1990) das Verfahren der „Mitwirkung des Volkes an der Rechtsprechung“, das als „Ehrenamt“ und „Bürgerpflicht“ (so \$ 1 ) ausgestaltet ist: Jede Gemeinde hat alle zwei Jahre eine Liste bestehend aus 0,5 Prozent aller in der Wählerevidenz verzeichneten Personen zu erstellen, aus der dann das Landesgericht LaienrichterInnen auslost. Grundsätzlich kann jede/r StaatsbürgerIn zwischen 25 und 65 Jahren auf dieser Liste stehen. Ausgeschlossen sind allerdings Personen mit körperlichen und geistigen Behinderungen, bestimmten Vorstrafen und jene mit mangelnden Deutschkenntnissen. Ausgenommen sind zudem der Bundespräsident, die Mitglieder von Bundes- oder Landesregierungen, Geistliche, RichterInnen, NotarInnen, PolizistInnen sowie jene ÖsterreicherInnen, deren Hauptwohnsitz sich im Ausland befindet. ${ }^{3}$

Über das Auslosungsverfahren hält das Gesetz in $\$ 5$ fest, dass dieses so zu geschehen habe, „daß die Auswahl einer jeden in Betracht kommenden Person mit annähernd gleicher Wahrscheinlichkeit möglich ist. Sie hat entweder durch ein automationsunterstütztes Datenprogramm oder auf eine andere, willkürliche Beeinflussung ausschließende Weise zu erfolgen." Demnach entscheidet gar zwei Mal das Los, zuerst bei der Erstellung der Gemeindelisten, dann bei der Berufung durch das Landesgericht. Ein qualifizierter Losentscheid kommt bei Jugend- und Sexualstraftaten

3 Im Gegensatz zu Österreich werden in der Bundesrepublik Deutschland Schöffen nur gelost, wenn sich für dieses Ehrenamt nicht genügend Freiwillige bewerben. 
zum Einsatz: Bei Jugendstrafverfahren muss die Hälfte der LaienrichterInnen als LehrerIn, PädagogIn oder in der Jugendwohlfahrt tätig sein. Mindestens zwei von ihnen müssen das Geschlecht des/der Angeklagten haben. Bei Sexualstraftaten müssen mindestens zwei LaienrichterInnen das Geschlecht des/der Angeklagten und mindestens zwei das Geschlecht des Opfers haben.

Die Laiengerichtsbarkeit gerät immer wieder in Kritik, hat stets ihre Für- und GegensprecherInnen. Eine wissenschaftliche Bewertung der Hinzuziehung von Schöffen und Geschworenen zu bestimmten Bereichen der Gerichtsbarkeit ist allerdings nicht Gegenstand des vorliegenden Textes. Daher soll hier für weiterführende Debatten nur auf den Fall Taxquet gegen Belgien (Urteil vom 16.11.2010, Bsw. 926/05) hingewiesen werden, worin der Europäische Gerichtshof für Menschenrechte keinen Anlass sah, die Laiengerichtsbarkeit insgesamt in Frage zu stellen (Decaigny 2010).

Für die gegenständliche Analyse ist vielmehr von Interesse, wie und warum das aleatorische Verfahren im Laufe der Entwicklung moderner Staatlichkeit einzig im Rechtssystem überdauerte. In der athenischen Polis war das Volksgericht, bestehend aus 6.00o Bürgern, gelost worden, aber auch andere, v.a. Verwaltungsorgane, wie der Rat der 500, der die politischen Geschicke lenkte. So exklusiv der Kreis, aus dem damals gelost wurde - Schätzungen reichen von zehn bis 25 Prozent der EinwohnerInnen - auch war, innerhalb dieses Zirkels war die Chance auf ein Amt für alle gleich. In der Neuzeit scheuten republikanische Gründerväter wie Madison allerdings diese grundsätzliche Gleichheit und setzten stattdessen auf Wahlen. Einzig im Rechtswesen blieb das Losverfahren bruchstückhaft erhalten. Da das US-amerikanische Jurysystem auf dem Argument der Demokratie breit diskutiert wurde und über Beobachter wie Alexis de Tocqueville auch die europäische Diskussion beeinflusste, steht es nun im Zentrum der historischen Betrachtung.

Die neue US-Demokratie allein auf Wahlen zu begründen und das Losverfahren auszuschließen, kritisierten alsbald die Anti-Federalists, jedoch nur für den Bereich der Gerichtsbarkeit, nicht für die allgemeine Führerauslese. Sie bemängelten den Verfassungstext, weil er nicht genügend Garantien für das Laiengerichtssystem in Zivilprozessen enthielt: „Für sie war die Institution der Jury eine Grundfeste republikanischer Demokratie. Danach musste man sich eine freie Republik aus zwei Strängen der Bürgerbeteiligung denken. Der eine Strang findet sich in der Ver- abschiedung von Gesetzen in einer Legislative, in die die Bürger ihre Repräsentanten wählen und mit ihnen in engem Kontakt bleiben; der andere Strang ist die Administration von Gesetzen, wozu nach damaligem Verständnis neben der Politik auf kommunaler Ebene auch die Gerichtsbarkeit gezählt wurde, da in Prozessen die Gesetze gleichsam , angewendet' würden“, hält Hubertus Buchstein (2009: 345f.) fest.

Die im modernen Staatswesen fortschreitende Gewaltentrennung brachte auch eine Aufspaltung der Begründung von Ämtern. Während die Legislative fortan gewählt wurde, hielt in der Exekutive das meritokratische Prinzip Einzug und bloß in der Judikative verblieben Spuren des Losverfahrens. Dadurch wurde das demokratische Prinzip der Gleichheit immer mehr in den Hintergrund gedrängt und durch ein moralisches Leistungssystem („Verdienst“, „Tugend“, „Weisheit“) ersetzt, das auf Basis ungleicher gesellschaftlicher Ausgangsbedingungen jedoch höchst elitär bleiben musste. Jahre später erkannte der österreichische Rechtswissenschafter Hans Kelsen in der Gewaltentrennung eine politische Absicht, um die breite Demokratisierung des Staates zu verhindern. Gemäß der Lehre vom Stufenbau der Rechtsordnung sind für ihn die drei Gewalten lediglich verschiedene Stadien der Rechtskonkretisierung. Somit ist auch die Gesetzgebung eine Art Vollziehung, nämlich Vollziehung der Verfassung, und „(z)wischen dem politischen Charakter der Gesetzgebung und dem der Justiz besteht nur eine quantitative, keine qualitative Differenz" (Kelsen 1930-31: 586). Zu dieser Einsicht war Kelsen insbesondere im Streit mit Carl Schmitt um die Frage, wer Hüter der Verfassung sei, gekommen. War für Schmitt der Reichspräsident als vom Volk gewählte unabhängige Instanz der Verfassungshüter, so verstand Kelsen diese Aufgabe pluralistisch. Nicht ein Präsident, auch nicht der Verfassungsgerichtshof (VfGH) sei allein Hüter der Verfassung, sondern in einer pluralistischen Demokratie sind ebenso das Parlament und die Verwaltung, ja jede/r einzelne BürgerIn zum Schutz der Verfassung aufgerufen (Ehs 2017).

Das sahen auch schon die Anti-Federalists so und bestanden aufgrund eines pluralistischen Verständnisses von Verfassungsschutz auf dem Einbezug der Bürger in die Juries: „Their situation as jurors and representatives, enables them to acquire information and knowledge in the affairs and government of the society, and to come forward, in turn, as the centinels and guardians of each other" (Federal Farmer IV, 1789, zit. nach Buchstein 2009: 346). Die Jury war gemäß 
diesem Konzept ein zentrales Instrument der Gesetzeskontrolle durch die Bürger und zugleich ein wichtiger Ort bürgerschaftlicher Partizipation. Entsprechend fand die Laiengerichtsbarkeit 1791 Eingang in die Bill of Rights. Als Alexis de Tocqueville die USA besuchte, wollte er in der Jury gar die Bedeutung für das Florieren der amerikanischen Demokratie erkennen. Er sah darin ,in erster Linie eine politische Einrichtung“, „eine Form der Volkssouveränität" (Tocqueville 1835: 409). Zudem hob er ihren edukativen Aspekt hervor, der die Bürger durch die Praxis mit den Gesetzen erst vertraut macht, und pries die soziale Durchmischung. Denn das Zufallsprinzip helfe, dass alle gesellschaftlichen Rollen und Positionen Beachtung fänden. Nur so könne auch ein Mann der Unterschicht als Richter tätig sein und damit seine Rolle als Bürger ausfüllen.

Tocqueville malte jedoch ein allzu schönes Bild; die Realität der Juries sah schon bald anders aus. Um den verachteten Pöbel fernzuhalten, losten die gewählten Politiker und ausgebildeten Richter die Laien nämlich nicht aus allen Wahlberechtigten, sondern aus einem Pool zuvor sorgfältig von ihnen ausgewählter Bürger, die charakterlich und religiös für fähig erachtet wurden. Und je weiter das allgemeine Wahlrecht auf dem Papier voranschritt, desto mehr Diskriminierungen wurden dort eingezogen, wo das Losverfahren echte Gleichheit hätte herstellen können. USA-weit setzte sich im 19. Jahrhundert die Elitenjury nach dem Key-Men-System durch. Key Men waren angesehene, meist wohlhabende Männer, die auf die Juryliste gesetzt wurden, wodurch die Jury bald nur noch aus einer kleinen, weißen Oberschicht bestand. Hubertus Buchstein gibt zu bedenken: „Die historische Forschungsliteratur über diese Epoche der amerikanischen Geschichte ist voll von skandalösen Prozessen, in denen weiße Geschworene über farbige Angeklagte zu Gericht saßen und zu Höchststrafen verurteilten“ (Buchstein 2009: 351). Erst der US Supreme Court führte allmählich wieder den Aspekt der demokratischen Gleichheit, den das Losverfahren ja verbürgen sollte, zurück ins Jurysystem. In den Urteilen Strauder v. West Virginia (1880), Smith v. Texas (1940) und Taylor v. Louisiana (1975) arbeitete er Schritt für Schritt das demokratische Prinzip der Gleichheit heraus: Die Jury müsse ein „body truly representative of the community" bilden und in ihr sollten alle ethnischen und sozialen Gruppen vertreten sein, die zur unmittelbaren Lebensumwelt des/der Angeklagten gehören. Im Mittelpunkt der Argumentation stand zuletzt vor allem die antidiskriminatorische Wirkung. Der blinde Mechanismus des Losens ermögliche keine Diskrimi- nierungen aufgrund von askriptiven Merkmalen wie „Tugend“ oder „Verdienst“ (Buchstein 2009: 356).

\section{Ausweitung des Losverfahrens im Rechtswesen - und darüber hinaus}

War das Losverfahren der Laiengerichtsbarkeit lange Zeit die einzige aleatorische Maßnahme im Rechtswesen, gibt es aktuell Überlegungen, es auch auf die BerufsrichterInnenernennung anzuwenden. Zurzeit wirbt etwa die Schweizer Justizinitiative für eine Auslosung der BundesrichterInnen. Statt der Bundesversammlung soll künftig ein Losentscheid das Bundesgericht personell besetzen. Die Initiative kritisiert die Parteinähe oder gar Parteimitgliedschaft, die BundesrichterInnen im aktuellen System abverlangt wird; zusammen mit der möglichen Wiederwahl nach sechs Jahren würde dies die Unabhängigkeit beeinflussen. Wissenschaftlich federführend ist der ehemalige Tessiner Kantonsrat und Politologe Nenad Stojanović von der Universität Genf. Er begründet den Einsatz des Losverfahrens im Justizbereich abermals mit dem Gleichheitsprinzip und möchte an die Schweizer Kantone gegen Ende des 18. Jahrhunderts anknüpfen, als das Losverfahren angewandt wurde, um die Regierenden auszuwählen. Dieser Idee nach würde eine Fachkommission sämtliche KandidatInnen für den Posten zuerst auf ihre Eignung (Absolvierung des Rechtsstudiums, Praxiserfahrung und dergleichen) prüfen, danach würde das Los - und eben nicht Parteinähe - entscheiden, wer den Job erhält. Somit käme das Prinzip der Chancengleichheit für diejenigen zum Zug, die über die erforderlichen Kompetenzen verfügen (Stojanović 2018).

Diese schweizerische Initiative aufgreifend brachte ich den Gedanken in die Debatte, auch in Österreich HöchstrichterInnen durch Losentscheid zu bestellen. Anlässlich der Kontroverse um die von der Bundesregierung als neue EuGH-Richterin nominierte, jedoch vom Artikel-255-Ausschuss abgelehnte Katharina Pabel, schlug ich vor, das Losverfahren auch auf diesen Bereich zu übertragen. Um demokratische Gleichheit zu gewährleisten und die Parteipolitik einzuhegen, sollte bei der nächsten RichterInnenbestellung für den EuGH ebenso wie für den VfGH nach Prüfung durch eine Fachkommission - gemäß der vom Artikel255-Ausschuss festgelegten Kriterien (von Bogdandy/ Krenn 2015) - das Los zum Zug kommen (Ehs 2018).

Jene Überlegungen und Initiativen beruhen einerseits auf dem Wunsch, parteipolitische und/oder allzu persönliche Erwägungen bei der Wahl unabhängiger 
und unparteiisch agierender RichterInnen außen vor $\mathrm{zu}$ lassen, andererseits stellen sie auf den demokratischen Aspekt der Gleichheit ab. Wie schon in der athenischen Polis sollte kein/e RichterIn das Amt erhalten, weil er/sie besonders für sich geworben hat oder sich jemand einen persönlichen Vorteil versprach. Da es in diesem System auch keine Wiederwahl gäbe, sondern abermals das Los entschiede, würden RichterInnen noch unabhängiger, d.h. ohne Blick auf ihre Karriere urteilen können.

Diese jüngsten Überlegungen, das Losverfahren nicht nur in der Laiengerichtsbarkeit anzuwenden, sondern auf andere Bereiche des Rechtswesens auszudehnen, korrespondieren mit einer allgemeinen Wiederentdeckung des aleatorischen Prinzips. Als Maßnahme gegen die Krise der repräsentativen Demokratie und eine wahrgenommene Politikverdrossenheit kommt die Lotterie seit einigen Jahren wieder vermehrt zum Einsatz (Piepenbrink 2013). Am bekanntesten ist wohl die irische Citizens' Assembly (BürgerInnenversammlung, anfänglich als Constitutional Convention zusammengetreten): Die politischen Institutionen der Republik Irland hatten durch die Finanzkrise 2008 bei vielen BürgerInnen einen immensen Vertrauensverlust erlebt (O’Sullivan/Healy/Breen 2014). Um diesem entgegenzutreten, entschlossen sich Parlament und Regierung $2011 \mathrm{zu}$ einem Demokratieexperiment. Zunächst 66, später 99 EinwohnerInnen Irlands wurden per Losverfahren ermittelt, um an mehreren Wochenenden unter Begleitung eines ModeratorInnenteams in Beratungen und ExpertInnenhearings Politikempfehlungen an die Regierung abzugeben. Die Constitutional Convention und nun die Citizens' Assembly tag(t)en unter der Prämisse, dass BürgerInnen gemeinsam in der Lage seien, zukunftsweisende Politikentscheidungen zu treffen. Auf diese Weise führte Irland 2015 per Verfassungsänderung die Ehe für alle ein, lockerte 2018 das strikte Abtreibungsverbot und debattierte grundsätzliche Fragen wie etwa den Umgang mit einer alternden Gesellschaft oder Irlands Beitrag zur Bekämpfung der Erderwärmung.

Ausgangspunkt dieses deliberativen Prozesses war die Lotterie: Ein Forschungsinstitut war beauftragt, mittels qualifizierter Zufallsauswahl einen irischen Minipopulus für die BürgerInnenversammlung $\mathrm{zu}$ erstellen; eine ausgewogene Mischung von Altersgruppen, Bildungshintergrund und sozioökonomischer Stellung, annähernde Geschlechterparität sowie Berücksichtigung von Migrationshintergrund und regionaler Diversität. Die somit bereinigte Zufallsaus- wahl garantierte, dass nicht - wie etwa bei direktdemokratischen und zivilgesellschaftlichen Instrumenten üblich - Männer Frauen majorisieren oder bildungsund einkommensstarke Gruppen in der Überzahl sind, während Menschen mit Migrationshintergrund fehlen. Wie nachfolgende wissenschaftliche Untersuchungen zeigten, erhöhte die Einbeziehung gewöhnlicher BürgerInnen in den Gesetzgebungsprozess sowie das transparente Verfahren das Vertrauen aller EinwohnerInnen Irlands in die Politik. Aufgrund des Erfolges sind Citizens' Assemblies nunmehr fixer Bestandteil der irischen Politikgestaltung (dazu affirmativ Narval 2018; kritischer Carolan 2015).

Was Irland erstmals auf nationalstaatlicher Ebene unternahm, kennt auch Österreich im Kleinen: Seit 2006 organisiert das beim Amt der Vorarlberger Landesregierung angesiedelte Büro für Zukunftsfragen Bürgerräte als partizipative Ergänzung der repräsentativen Demokratie. Diese werden auf Gemeinde-, Regionalund Landesebene durchgeführt und sind bereits fixer Bestandteil der Politikgestaltung im Ländle. Seit 2013 ist dieses Instrument der partizipativen Demokratie auch in der Landesverfassung verankert und kann mittels 1.000 Unterschriften auch von BürgerInnen selbst initiiert werden. An Bürgerräten teilnahmeberechtigt sind alle in Vorarlberg wohnhaften Menschen, die per Zufallsauswahl aus dem Melderegister eingeladen werden. Der Bürgerrat formuliert Lösungsvorschläge, die an die Landesregierung weitergegeben werden. Diese muss sich mit den Ergebnissen auseinandersetzen; eine Verpflichtung zur Umsetzung der Vorschläge gibt es jedoch nicht.

Beispielgebend für die Vorarlberger Bürgerräte wie auch für die irische BürgerInnenversammlung war die Citizens' Assembly on Electoral Reform in der kanadischen Provinz British Columbia, die in den Jahren 2003 bis 2005 mit 161 gelosten BürgerInnen ein neues Wahlrecht ausarbeitete (Warren/Pearse 2008). Lange Jahre vor dem aktuellen Hype um das Losverfahren bildete Peter C. Dienels Planungszelle eine Vorläuferin. Der deutsche Soziologe hatte in den 1970er-Jahren insbesondere für die BürgerInnenbeteiligung bei größeren Bauvorhaben einen Prozess entwickelt, bei dem pro Planungszelle 25 im Zufallsverfahren ausgewählte Personen ab 16 Jahren für ca. eine Woche von ihren arbeitsalltäglichen Verpflichtungen freigestellt werden, um Lösungsvorschläge für ein vorgegebenes Planungsproblem zu erarbeiten (Dienel 2002). Etwa zur gleichen Zeit konzipierte Ned Crosby in den USA ebenso für Beteiligungsverfahren die Citizens' Jury, und zwar 
angelehnt an die gleichnamige Laiengerichtsbarkeit - womit sich der Kreis meiner historischen Umschau schließt. ${ }^{4}$

\section{Demokratiepolitischer Hintergrund: Input-/ Throughput-/Output-Legitimation}

Wie der geschichtliche Überblick zeigte, stand das Losverfahren bei der politischen Ämterbesetzung in Konkurrenz zur Wahl und galt lange Zeit als das demokratischere Mittel. Die Wahl wurde als aristokratisch betrachtet, weil sie den Gleichheitsanforderungen nicht genügte. Dennoch erfolgte die weltweite Ausbreitung der Demokratie in den vergangenen zwei Jahrhunderten aufgrund von Wahlen und die beiden Seiten der Demokratie - Freiheit und Gleichheit - wurden mit unterschiedlichen Maßnahmen und variierender Intensität verfolgt. Chantal Mouffe (2008) spricht im Zusammenhang mit diesen beiden Seiten vom „demokratischen Paradox“, das der liberalen Demokratie westlicher Prägung innewohne. Im Zuge der neoliberalen Hegemonie der vergangenen 40 Jahre sei die liberale Komponente übermächtig, hingegen die Infrastruktur des nach dem Zweiten Weltkrieg errichteten klassenbasierten Regimes politischer Repräsentation - also gewerkschaftliche Organisation, industriepolitische Arrangements, Wohlfahrtsstaat, öffentlicher Sektor, Diskurse des „sozialen Ausgleich““ - sukzessive ausgezehrt worden. Während die Freiheit heute überbetont sei, komme die Gleichheit zu kurz. Diese Entwicklung blockiere nun die politische Repräsentation vor allem der subalternen Interessen strukturell, was zu sinkender Wahlbeteiligung, Politikverdrossenheit und letztlich der Gefahr des autoritären Populismus führe (Gallas/Nowak 2011). Aufgrund der Vernachlässigung der demokratischen Gleichheit bleibt jene leere Hülle von Demokratie übrig, die Colin Crouch nach Jacques Rancière als „Postdemokratie“ bezeichnet.

Seit der multiplen Krise von 2008 und den darauf folgenden Demokratiebewegungen - von Occupy Wall Street über den Movimiento 15-M und die Indignados bis $\mathrm{zu}$ entsprechenden Parteigründungen wie Podemos und Syriza und Parteierneuerungen wie Labour unter Jeremy Corbyn - rückt das Thema der Gleichheit gemeinsam mit der Sozialen Frage wieder ins Zentrum der politischen Auseinandersetzung. Und mit ihm kam das Losverfahren zurück. Die Republik Irland

$4 \quad \mathrm{Zu}$ einer Typologie der politischen Lotterien siehe Baron 2013. trat mit der Citizens' Assembly gleichsam die Flucht in die Demokratie an und legte dem BürgerInnenbeteiligungsverfahren die Lotterie zugrunde, um wieder Vertrauen in ihre politischen Institutionen generieren zu können. Denn wie Untersuchungen ergaben, vertrauen die BürgerInnen angesichts großer politischer Krisen eher einander als den staatlichen Behörden. Die Vorarlberger Bürgerräte wiederum sehen die gelosten Gruppen zudem als Mittel gegen Populismus. Denn wenn Menschen ihre Echokammern verlassen und über soziale, ökonomische und Altersgrenzen hinweg miteinander ins (moderierte) Gespräch kommen, verlieren populistische Vorschläge im Lauf der Diskussionen deutlich an Boden (Fehrenbach 2017).

Die qualifizierte Zufallsauswahl des Loses trägt dazu bei, die bei selbstinitiativer politischer Beteiligung stets auftretende sozio-ökonomische Kluft sowie den Gendergap zu schließen, indem sie Menschen in den politischen Prozess bringt, die nicht ohnehin bereits AktivbürgerInnen sind. Der Politikwissenschafter Armin Schäfer (2015) weist aufgrund internationaler Studien nämlich eindringlich darauf hin, dass sowohl die Wahlbeteiligung als auch weitere Möglichkeiten der politischen Partizipation nicht von allen sozialen Schichten gleich genutzt werden. Menschen in prekären Lagen, die über weniger Ressourcen wie Einkommen oder Bildung verfügen, gehen nicht nur seltener wählen, sondern sind auch seltener in BürgerInneninitiativen, bei politischen Versammlungen oder ähnlichen Aspekten bürgerschaftlichen Engagements zu finden. Diese soziale Schieflage politischer Partizipation sei laut Schäfer (2013: 46) mit der seit einigen Jahren wieder wachsenden sozialen Ungleichheit gestiegen: „Für die Demokratie besteht die Gefahr einer niedrigen und sozial ungleichen Wahlbeteiligung darin, dass die Politik sich an den sozial Bessergestellten orientieren könnte, die nicht nur weiterhin wählen, sondern auch andere Wege nutzen, ihre Anliegen zur Sprache zu bringen, während sozial Benachteiligte weder das Eine noch das Andere in gleichem Umfang tun." Mit "anderen Wegen“ sind Instrumente wie BürgerInnenpetitionen oder die in Österreich laut aktuellem Regierungsprogramm zur Diskussion stehenden von unten zu initiierenden Volksabstimmungen gemeint.

Die Entwicklung der wachsenden sozialen Ungleichheit bedroht den Gleichheitsaspekt der Demokratie, der nach der gleichen Berücksichtigung aller Interessen verlangt. Arme, prekär Beschäftigte und formal Geringgebildete haben im gegenwärtigen System nicht dieselbe Chance, dass ihre Anliegen im 
politischen Prozess gehört und umgesetzt werden, wie zuletzt Lea Elsässer in ihrer Responsivitätsstudie zur deutschen Sozial- und Arbeitsmarktpolitik im Zeitraum von 1980 bis 2013 eindrücklich darlegte (Elsässer 2018). Soziale Ungleichheit untergräbt letztlich das Vertrauen in die repräsentative Demokratie und ihre Problemlösungsfähigkeit; übrig bleiben „dysfunktionale Gesellschaften“ (Wilkinson/Pickett 2009, Kap. 13) mit Neigung zu autoritärem Populismus. ${ }^{5}$

Um die soziale Ungleichheit und ihre politischen Folgen einzudämmen, idealerweise auf ihre Überwindung hinzuarbeiten, gibt es einerseits die großen Antworten in der Sozial-, Arbeitsmarkt- und Bildungspolitik, die darauf abzielen der „Abstiegsgesellschaft“ (Nachtwey 2016) entgegenzuwirken; und andererseits gibt es die kleineren, nicht minder wichtigen Antworten, die den Einbezug aller BürgerInnen in den politischen Prozess befördern: Mittels qualifizierter Zufallsauswahl geloste Bürgerräte könnten Menschen am Deliberationsprozess beteiligen, die sonst aufgrund ihres sozioökonomischen Status, ihres Bildungsgrades oder ihrer Herkunft ungehört bleiben. Alexander Guerrero (2014) nennt „the failure of responsiveness and the failure of good governance" die beiden größten Systemfehler der zeitgenössischen Demokratie und führt explizit das Losverfahren als Gegenmittel an.

Neben jenen genannten Vorteilen für die Inputund Throughput-Legitimation durch das Losverfahren werden stets auch Output-Faktoren diskutiert. Im Vordergrund stehen hierbei nicht-populistische Entscheidungen, die dann gefällt werden können, wenn der Beratungsprozess im Vorfeld stärker demokratisiert ist und insbesondere wenn - wie etwa im Falle Irlands - Referenden von Bürgerräten begleitet werden, die die öffentliche Deliberation befördern. Der Politologe James Fishkin, der seit über 30 Jahren deliberative Prozesse gestaltet und wissenschaftlich begleitet, entwickelte hinsichtlich des Einbezugs von gelosten Bürgerräten in die repräsentative Demokratie die Schlussfolgerung, dass ,this is what the people

5 Siehe hierzu die Studie von SORA und dem Verein zur wissenschaftlichen Aufarbeitung von Zeitgeschichte, die erhoben, dass immer mehr Befragte der Aussage, man „sollte einen starken Führer haben, der sich nicht um ein Parlament und Wahlen kümmern muss" zustimmen. Sie führen diesen Anstieg auf ökonomische Unsicherheiten und steigende soziale Ungleichheiten zurück: https://www.sora.at/nc/newspresse/archiv/news-einzelansicht/news/schon-43-fuer-starken-mann-776.html [12.02.2019]. would think about the issue under good conditions for thinking about it" (Fishkin 2018: 374). Es geht hinsichtlich der Output-Legitimation von gelosten Bürgerräten demnach darum, Strukturen zu schaffen, durch die BürgerInnen von der raw opinion, also vom bloßen Bauchgefühl, zur informed opinion gelangen.

Nicht zuletzt in Hinblick auf die aktuelle Skepsis gegenüber ExpertInnen oder Wissenschaft im Allgemeinen (Stichwort Erderwärmung, ImpfgegnerInnen etc.) sowie auf fake news als teils wahlentscheidender Faktor sehen PolitikwissenschafterInnen die Instrumente partizipativer Demokratie als Möglichkeit, die Kluft zwischen BürgerInnen und wissenschaftlicher Evidenz zu überwinden (Elstub et al 2018; Breckon et al 2019). Auf dem Losverfahren basierende Maßnahmen wie Bürgerräte dienen der Demokratie somit mehrfach im Sinne der politischen Integration und insbesonders des schon von Tocqueville gepriesenen edukativen Aspekts.

\section{Aktuelle und künftige Anwendungen des Losverfahrens}

Der aktuelle Hype um geloste Bürgerräte steht mit der Krise des Vertrauens in die Problemlösungsfähigkeit der (repräsentativen) Demokratie in Zusammenhang, die mit der Wirtschafts- und Finanzkrise seit 2008 breitere öffentliche Diskussion erfährt. Zwar hatte bereits Josiah Ober (1989) Denkanstöße aus dem klassischen Athen für die moderne Demokratie gegeben und antike Verfahren mit heutigen Fragestellungen kontrastiert; doch Bekanntheit erlangte das Verfahren im deutschen Sprachraum erst mit den Werken von Buchstein (2009) und populärwissenschaftlich gefasst durch Van Reybrouck (2016). Das heute für die Demokratie angewandte und propagierte Losverfahren beruht zwar ideell auf dem antiken Prinzip, unterscheidet sich jedoch in der Praxis durch die repräsentative Stichprobe, die den Minipopulus zum Ziel hat, worauf insbesondere Sintomer (2016) hinweist.

Ob nun in Irland, Vorarlberg oder British Columbia - im Fokus der partizipativen Demokratie steht nicht wie bei vielen zeitgenössischen Forderungen nach mehr direkter Demokratie der Moment des Plebiszits, also nicht die Abstimmung, sondern die politische Deliberation. Dem Losverfahren kommt hierbei die Aufgabe zu, das politische Amt des Beraters beziehungsweise der Beraterin auf Zeit zu vergeben, im Grunde den Lobbyismus zu demokratisieren, indem er ihn auch auf Menschen ausweitet, die sonst oft nicht 
einmal (mehr) durch Wahlen politischen Einfluss nehmen (können). ${ }^{6}$

Neben der Sozialen Frage und der Geschlechtergerechtigkeit gerät auch immer mehr die Generationengerechtigkeit in den Blick der BefürworterInnen dieses Instruments. Denn in der Kategorie Alter ist die demokratische Teilnahmekluft noch größer als zwischen Männern und Frauen. Entsprechend gibt es mittlerweile zahlreiche Jugendinitiativen, die sich für das Losverfahren stark machen (Rieg 2013). Zuletzt verwirklichte der hauptsächlich von jungen Menschen getragene Verein mehr als wählen für den ersten Frankfurter Demokratiekonvent den qualitativen Minipopulus derart, dass von den 50 TeilnehmerInnen zwar zwei Drittel aus dem Melderegister gelost, aber ein Drittel mit Hilfe von Vereinen ausgewählt wurden, um jene Menschen zu erreichen, die in der Stadtpolitik unterrepräsentiert sind, womit explizit MigrantInnen und Jugendliche gemeint waren.

Bislang stellten geloste Bürgerräte selbst dort, wo sie im Rechtsbestand als Instrumente der partizipativen Demokratie festgehalten sind (so etwa in der Vorarlberger und Salzburger Landesverfassung), punktuelle Ereignisse mit wechselnden TeilnehmerInnen dar. Doch 2019 scheint das Jahr der Institutionalisierungen zu sein: Ende Jänner beschloss die Stadt Madrid den ersten permanenten Bürgerrat der neueren europäischen Geschichte, den Observatorio de la Ciudad. 49 per Zufallslos ermittelte MadrilenInnen bekleiden ab dem Frühjahr ein Jahr lang ihr Amt und beraten die Stadtregierung. 2020 werden neue BürgerrätInnen gelost. Das Parlament der deutschsprachigen Gemeinschaft Belgiens zog Ende Feber nach und richtet zurzeit einen 24 Personen umfassenden gelosten Bürgerrat ein, der die Themen für eine ebenfalls geloste BürgerInnenversammlung festlegen und in der politischen Umsetzung mit der Regierung zusammenarbeiten wird. An der Konzeption des sogenannten Ostbelgienmodells war u.a. David Van Reybrouck federführend beteiligt.

Jene Institutionalisierungen entsprechen der von Patrizia Nanz und Claus Leggewie als „vierte Gewalt“ konzipierten Konsultative (Nanz/Leggewie 2016), die als permanente demokratische BürgerInnenversammlung eine dringend notwendige Repolitisierung des

6 Da Bürgerräte meist nicht auf die Staats- sondern allein auf die Wohnbürgerschaft abstellen, verwirklichen sie auch in dieser Hinsicht mehr Demokratie (Zum Ausschluss von der Demokratie aufgrund des Staatsbürgschaftserfordernisses siehe näher Ehs 2018a).
Beratungsprozesses bilde. Außerdem korrespondieren sie mit den in der Wissenschaft (zuletzt Gastil/Wright 2018) schon länger getätigten Überlegungen, die zweite Kammer der Landes- und/oder Regionalparlamente durch geloste BürgerInnenversammlungen zu ersetzen beziehungsweise Einkammernsysteme um eine solche zweite Kammer zu ergänzen. Für Österreich würde dies auf Nationalstaatsebene bedeuten, den Bundesrat als Bürgerrat neu zu gründen. Seine Mitglieder wären nicht länger VertreterInnen der Bundesländerparteien, sondern per Los ermittelte BürgerInnen, die ein Jahr lang den Nationalrat in dessen Gesetzgebungstätigkeit beraten. Ähnliches wäre für die neun Landesparlamente zu konzipieren. James Fishkin brachte neulich in die Diskussion ein, diese Idee mit dem von ihm bereits seit 1988 weltweit erprobten Deliberative Polling zu verbinden (Fishkin 2018). John Gastil und Erik Olin Wright widmen jenen Gedanken einen demnächst erscheinenden Sammelband (Gastil/Wright 2019).

Einen kleinen Schritt in diese Richtung hatte das österreichische Parlament im Rahmen der 2015 abgehaltenen Enquetekommission Zur Stärkung der Demokratie getan. Zur Teilnahme mit Rederecht (allerdings ohne Stimmrecht) waren erstmals auch acht BürgervertreterInnen eingeladen. Für einen Platz hatte man sich bewerben können. Aus den insgesamt 1.200 Bewerbungen wurden schließlich vier Frauen und vier Männer, davon die Hälfte unter und die andere Hälfte über 35 Jahren, ausgelost. Eine weitere Anwendung des Losverfahrens wurde 2018 von der SPÖ im Rahmen der Erarbeitung eines neuen Parteiprogramms unternommen. Um nicht nur jene zu hören, die sich ohnehin bereits aktiv in der Partei einbringen, wurden unter sämtlichen Mitgliedern Plätze für die Teilnahme an themenspezifischen Diskussionstagen verlost. Die sogenannten Mitgliederräte wurden durch eine qualifizierte Zufallsauswahl besetzt, die Gender- und Altersbalance, regionale Verteilung sowie unterschiedliche Dauer der Parteimitgliedschaft berücksichtigte.

In der Schweiz wird demnächst mit dem Losverfahren als Grundlage einer nicht-populistischen direkten Demokratie experimentiert. Der bereits erwähnte Politikwissenschafter Stojanović möchte die schweizerische Demokratie um ein BürgerInnenkomitee ergänzen. Dabei sollen im Vorfeld eines Referendums geloste BürgerInnen über fünf Tage hinweg gemeinsam mit ExpertInnen über den Gesetzestext diskutieren; ihr Gutachten ergeht gemeinsam mit dem üblichen Abstimmungsbüchlein an alle StimmbürgerInnen. Wie bereits bei der irischen Citizens' Assembly 
soll durch den Einbezug gewöhnlicher BürgerInnen auch das Vertrauen in die politischen Institutionen gestärkt werden. Im Vordergrund steht abermals das Prinzip der Gleichheit: „Dank der Auslosung wird das Prinzip der Gleichheit stärker und bezieht Menschen mit ein, die in der Regel nicht gleichberechtigt sind. Gleichzeitig ermöglichten diese Diskussionsforen, der Öffentlichkeit und den Behörden neue Informationen zukommen zu lassen“, so Alice el-Wakil, Mitarbeiterin dieses Experiments (2018).

Obwohl es sich bei jenen Bürgerräten und zweiten Kammern juristisch gesehen um soft law handelt, weil sie eben nur „Rat“ geben, aber nicht entscheiden, entfalten sie ihre Wirkung in der Input- und ThroughputLegitimation des politischen Systems. Die bisherige Praxis zeigt, dass geloste Bürgerräte dann auf diesen beiden Ebenen erfolgreich sind, wenn erstens die Auswahl der BürgerrätInnen nach einem transparenten Verfahren erfolgt und zweitens gewährleistet ist, dass Legislative und Exekutive Rechenschaft ablegen, ob und welche Wirkung die Ergebnisse der Beratungen auf den weiteren politischen Prozess haben.

\section{Conclusio}

Die Gelingensbedingungen und konkreten Ausgestaltungen der Verfahren partizipativer Demokratie sind mittlerweile nicht nur weltweit vielfach praktisch erprobt, sondern auch wissenschaftlich kritisch erörtert: Hervorzuheben ist etwa Caroline W. Lees Studie Do-It-Yourself Democracy (2014), die eine umfassende, auf allgemeiner Kapitalismuskritik beruhende Abhandlung über die sogenannte „Demokratieindustrie“ vorlegte und darin auch auf zahlreiche Fallstricke der deliberativen Demokratie hinwies. Weiters sind allen voran James Fishkin, John Gastil und Erik Olin Wright und im deutschen Sprachraum Hubertus Buchstein seit Jahren Experten auf dem Gebiet des politischen Losverfahrens. Doch während nicht nur international, sondern auch in Österreich mit verschiedenen Instrumenten experimentiert wird, sind die wissenschaftlichen Untersuchungen hierzulande rar gesät. Abgesehen von Masterarbeiten (Oppold 2016; Toth 2017) und einigen hauseigenen Analysen der Landesregierung ist der Vorarlberger Bürgerrat nicht systematisch untersucht. Zwar betrachteten unlängst Peter Biegelbauer und Sandro Kappeller (2017) sechs lokale Entscheidungsfindungsprozesse, beurteilten die Möglichkeit zur demokratischen Mitgestaltung durch die BürgerInnen und erachteten alle Prozesse als gescheitert; das Losverfahren war allerdings in keiner der untersuchten Gemeinden zum Einsatz gekommen.

Für eine intensive Reflexion über den Einsatz von gelosten Minipopuli im Gesetzgebungsverfahren muss demnach auf internationale Studien zurückgegriffen werden. Fishkin (2018) meinte vor Kurzem, dass nach jahr(zehnt)elangem Ausprobieren verschiedenster deliberativer Instrumente nun die Zeit der Institutionalisierungen gekommen sei; und die jüngsten Ereignisse in Madrid und Ostbelgien geben ihm recht. Die aktuell in praxi noch größte Herausforderung beim aleatorischen Verfahren sieht er in Organisationsfragen. Denn ohne umfangreiche Bemühungen im Vorfeld und oft kostspielige Zusammenarbeit mit Forschungsinstituten sei der Minipopulus nicht zu verwirklichen. Setze man bloß auf die Ziehung aus dem Melderegister und dann Einladung per Amtsbrief, sei die Rücklaufquote der Eingeladenen oft viel zu gering, sodass die gewünschte politische Egalität schließlich nicht hergestellt werden kann. Tatsächlich verzeichneten z.B. die Vorarlberger Bürgerräte erst im Laufe der Jahre einen Anstieg von TeilnehmerInnen aus sozioökonomisch benachteiligten Schichten, lassen aber nach wie vor Menschen mit Migrationshintergrund vermissen.

Die Gelingensvoraussetzung des Losverfahrens im Sinne politischer Gleichheit beruht daher auf gründlicher Organisation und budgetärer Absicherung, wie sie etwa die Citizens' Assembly leistet, die erst startet, wenn der Minipopulus vollständig ist. Dafür ist es unerlässlich, dass die OrganisatorInnen alle möglichen Teilnahmehindernisse (wie etwa familiäre Sorgepflichten, körperliche Einschränkungen etc.) kennen und gemeinsam mit den ausgelosten BürgerInnen überwinden. Darüber hinaus dürfen den TeilnehmerInnen keinerlei finanzielle Nachteile entstehen, weshalb Reise- und Aufenthaltskosten zu übernehmen sind. Zudem erhalten BürgerrätInnen oft Taggeld und/ oder Honorare. Sind diese Voraussetzungen gegeben, gelingt es meist problemlos, den Minipopulus herzustellen, wie Sarah Allan und Rebecca McKee (2019) in ihrer laufenden Untersuchung belegen. Weiters ist anzumerken, dass auch die partizipative Demokratie eines Lernprozesses bedarf: Je länger und je öfter dieses Verfahren angewandt wird, desto größere Öffentlichkeit und Vertrauen erlangt es, was letztlich die Teilnahmebereitschaft der Eingeladenen erhöht.

Die oft sorgenvolle Frage jener, die zum ersten Mal mit dem Thema konfrontiert sind, ob die Teilnahme an gelosten Bürgerräten etwa ähnliche Pflichtqualität habe oder erlangen müsste wie in der Schöffen- und 
Geschworenengerichtsbarkeit, stellt sich demnach nur, wenn die politisch Verantwortlichen nicht bereit sind, genügend Organisationsmittel zur Verfügung zu stellen. Nur wer die Teilnahme nicht so niederschwellig und einladend wie möglich gestaltet, muss auf Druck und/oder gar Bußgelder setzen. Zur Orientierung in der Kostenfrage sei angemerkt: Die irische Constitutional Convention wurde mit $900.000 €$ ausgestattet; für die Citizens' Assemblies sind nunmehr 600.00o $€$ pro Jahr budgetiert, die jedoch regelmäßig überschritten werden. Das Parlament der deutschsprachigen Gemeinschaft Belgiens berechnet sein Modell - meines Erachtens zu knapp - zurzeit mit ca. $140.000 €$ pro Jahr. Daraus sind immerhin der/die ständige SekretärIn, Entschädigungen der BürgerInnen, Organisationsund Logistikkosten, Honorare der ExpertInnen und ModeratorInnen sowie die Kosten für das Losverfahren zu bestreiten.

Mein Text legte anhand eines historischen Überblicks dar, dass der Einsatz des Losverfahrens im politischen Prozess keineswegs neu ist, sondern auf den Gleichheitsüberlegungen der frühesten Demokratien beruht. War er in jüngerer Zeit jedoch auf den Bereich der Laiengerichtsbarkeit beschränkt, so erfährt er nun durch die mannigfach konstatierte Demokratiekrise gemeinsam mit der neuen Sozialen Frage ein Revival. Überall dort, wo das demokratische System heute als defizitär erlebt wird, weil es in Bezug auf die Geschlechter, sozialen Milieus oder den Migrationshintergrund nicht hinreichend repräsentativ ist, kann auf prozeduraler Ebene die Lotterie Abhilfe schaffen. Seitens vieler BefürworterInnen dieses Instruments besteht freilich die Hoffnung, dass sich die Gleichheit im Zugang zu politischer Beratung und Einflussnahme letztlich in entsprechende Politikinhalte übersetzt, die auch mehr substanzielle Gleichheit nach sich ziehen.

\section{Literatur}

Allan, S./McKee, R. (2019): Why do citizens' assemblies work? Online: The Constitution Unit at University College London, https://constitution-unit.com [28.02.2019].

Aristoteles (1991): Politik. Buch II. In: Schütrumpf, E. (Hg.): Aristoteles - Werke in deutscher Übersetzung. Band 9/II Politik - Buch II und III. Berlin, Boston: De Gruyter.

Baron, D. (2013): Politische Partizipation durch Losentscheid. Ein systematisierender Typologisierungsansatz. In: Nève, D. de/Olteanu, T. (Hg.): Politische Partizipation jenseits der Konventionen. Leverkusen: Barbara Budrich, 75-102.

Biegelbauer, P./Kappeller, S. (2017): Mitentscheiden oder Mitgestalten: Direkte Demokratie versus dialogorientierte Verfahren in lokalen Entscheidungsfindungsprozessen. SWS-Rundschau, 57 (1), 32-55.

Bogdandy, A. von/Krenn, Ch. (2015): Zur demokratischen Legitimation von Europas Richtern. Eine vergleichende Rekonstruktion der Richterauswahl zu EGMR und EuGH. Juristenzeitung, 11 (69), 529-584.

Breckon, J./Hopkins, A./Rickey, B. (2019): Evidence vs Democracy. How 'mini publics' can traverse the gap between citizens, experts and evidence. London: Alliance for Useful Evidence, https://www.alliance4usefulevidence.org/ [03.03.2019].

Buchstein, H. (2009): Demokratie und Lotterie. Das Los als politisches Entscheidungsinstrument von der Antike bis zur EU. Frankfurt am Main: Campus.

Burke, E. (1790/1987): Betrachtungen über die Französische Revolution. Zürich: Manesse.

Burnheim, J. (1987): Über Demokratie. Alternativen zum Parlamentarismus. Berlin: Wagenbach.

Carolan, E. (2015): Ireland's Constitutional Convention: Behind the hype about citizen-led constitutional change. International Journal of Constitutional Law, 13 (3), 733748.

Dahl, R. A. (1971): Polyarchy. Participation and Opposition. New Haven/London: Yale University Press.

Decaigny, T. (2010): ECtHR Taxquet v. Belgium. Can Juries Convict in Accordance with the European Convention on Human Rights? New Journal of European Criminal Law, 1 (1), 7-15.

Dienel, P. C. (2002): Die Planungszelle. Der Bürger als Chance. 5. Auflage. Wiesbaden: Springer VS.

Duxbury, N. (1999): Random Justice: On Lotteries and Legal Decision-Making. Oxford: UP.

Ehs, T. (2017): Das politische Element der Verfassungsgerichtsbarkeit. In: Özmen, E. (Hg.): Hans Kelsens Politische Philosophie. Tübingen: Mohr Siebeck, 133-147.

Ehs, T. (2018): Österreichs neue EuGH-Richterin: nominieren, prüfen, auslosen? Wiener Zeitung, 12.06.2018, 4.

Ehs, T. (2018a): Wien wählt (nicht). Demokratische Beteiligung 1918-2018. Perspektiven. Policy brief 6/2018 des Jahoda-Bauer-Instituts, Linz.

Ehs, T./Willroider, N. (2013): Parlamente unter Druck. Die Rückkehr des direktdemokratischen Marktplatzes. juridikum. Zeitschrift für Recht, Kritik, Gesellschaft, 1, 119129.

Elsässer, L. (2018): Wessen Stimme zählt? Soziale und politische Ungleichheit in Deutschland. Frankfurt a. M./New York: Campus.

Elstub, S./Johnson, I./Puttick, R./Wilkinson, M. (2018): Assessing the Potential of Mini-Publics to Promote Evidence Uptake in Social Policy and Practice. Newcastle: UP. 
Fehrenbach, G. (2017): Wir müssen lernen, heikle Fragen $z u$ stellen. Online: https://medium.samu.works/wirmüssen-lernen-heikle-fragen-zu-stellen-5ea3c77784ba [13.02.2019].

Fenazzi, S. (2018): Bei komplexen Entscheiden werden Bürger zu Beratern. Online: https://www.swissinfo.ch/ger/ direktedemokratie/oregon-modell_bei-komplexenentscheiden-werden-buerger-zu-beratern/4400o884 [12.11.2018].

Fishkin, J. (2018): Random Assemblies for Lawmaking? Prospects and Limits. Politics \& Society, 46 (3), 359-379.

Franklin, M. N. (2004): Voter turnout and the dynamics of electoral competition in established democracies since 1945. Cambridge: UP.

Gallas, A./Nowak, J. (2011): Die Krise der Demokratien: Wahlautokratie, Klassenpolitik mit leeren Händen, Kulturalisierung. Österreichische Zeitschrift für Politikwissenschaft, 40 (2), 139-153.

Gastil, J./Wright, E. O. (2018): Legislature by Lot: Envisioning Sortition within a Bicameral System. Politics \& Society, 46 (3), 303-330.

Gastil, J./Wright, E. O. (2019): Legislature by Lot. London: Verso.

Guerrero, A. (2014): Against Elections: The Lottocratic Alternative. Philosophy and Public Affairs, 42 (2), 135-178.

Kelsen, H. (1930/31): Wer soll Hüter der Verfassung sein? Die Justiz, 6, 576-628.

Lee, C. W. (2014): Do-It-Yourself Democracy. The Rise of the Public Engagement Industry. Oxford: UP.

Madison, J. (1787/1993): Federalist Paper Nr. 10. In: Hamilton, A./ Madison J./ Ray, J., Die Federalist Papers. Darmstadt: Wissenschaftliche Buchgesellschaft.

Manin, B. (2007): Kritik der repräsentativen Demokratie. Berlin: Matthes \& Seitz.

Montesquieu, Ch. de (1748/1965): Vom Geist der Gesetze, 2. Buch, 2. Kapitel. Stuttgart: Reclam.

Mouffe, Ch. (2008): Das demokratische Paradox. Wien: Turia+Kant.

Nachtwey, O. (2016): Die Abstiegsgesellschaft. Über das Aufbegehren in der regressiven Moderne. Berlin: Suhrkamp.

Nanz, P./Leggewie, C. (2016): Konsultative. Mehr Demokratie durch Bürgerbeteiligung. Berlin: Wagenbach.

Narval, Ph. (2018): Die freundliche Revolution. Wie wir gemeinsam die Demokratie retten. Wien: Molden.

Ober, J. (1989): Mass and Elite in Democratic Athens. Rhetoric, Ideology, and the Power of the People. Princeton: UP.

Oppold, D. (2016): Effekte deliberativer Demokratie am Beispiel der Bürgerräte in Vorarlberg. Masterarbeit an der Universität Konstanz.

O’Sullivan, S./Healy, A. E./Breen, M. J. (2014): Political Legitimacy in Ireland During Economic Crisis: Insights from the European Social Survey. Irish Political Studies, 29 (4), 547-572.

Piepenbrink, K. (2013): Losverfahren, Demokratie und politische Egalität: Das Losprinzip im klassischen Athen und seine Rezeption im aktuellen Demokratiediskurs. Antike und Abendland, 59, 17-31.

Rawls, J. (1971): A Theory of Justice. Cambridge: UP.

Reybrouck, D. Van (2016): Gegen Wahlen. Warum Abstimmen nicht demokratisch ist. Göttingen: Wallstein.

Rieg, T. (2013): Youth Citizens Jury. Erfahrungen mit einem neuen Modell für Jugendpartizipation. deutsche jugend, 6, 245-253.

Rilling, R. (2014): Thomas Piketty und das Märchen vom Gleichheitskapitalismus. Blätter für deutsche und internationale Politik, 11, 81-91.

Rousseau, J.-J (1762/1989): Vom Gesellschaftsvertrag oder Prinzipien des Staatsrechts. 4. Buch, 3. Kapitel. Berlin: Rütten \& Loening.

Schäfer, A. (2013): Wahlbeteiligung und Nichtwähler. Aus Politik und Zeitgeschichte, 48/49, 39-46.

Schäfer, A. (2015): Der Verlust politischer Gleichheit. Warum die sinkende Wahlbeteiligung der Demokratie schadet. Frankfurt a. M.: Campus.

Sintomer, Y. (2016): Das demokratische Experiment. Geschichte des Losverfahrens in der Politik von Athen bis heute. Berlin: Springer.

Stojanović, N. (2018): Das Losverfahren bringt Vorteile für die Demokratie. Online: www.justiz-initiative.ch [09.02.2019].

Taylor, C. (2007): From the Whole Citizen Body? The Sociology of Election and Lot in the Athenian Democracy. Hesperia, 76 (2), 323-345.

Tocqueville, A. de (1835/1976): Über die Demokratie in Ameri$k a$. Vol. I. München: dtv.

Toth, K. (2017): Public participation, sustainability and environmental awareness. Case study of the effect of wisdom councils on participants in the region Bregenzerwald. Masterarbeit an den Universitäten Kopenhagen (UCPH) und BOKU Wien.

Warren, M./Pearse, H. (Hg.) (2008): Designing Deliberative Democracy: The British Columbia Citizens' Assembly. Cambridge: UP.

Wilkinson, R./Pickett, K. (2009): The spirit level: Why more equal societies almost always do better. London: Penguin. 